\title{
DETERMINANTS OF FOLLOWING HEADWAY IN CONGESTED TRAFFIC
}

\author{
TRANSPORTATION RESEARCH PART F - 12(2), 131-142
}

\author{
Mark Brackstone \\ (*Egis Mobilite UK Ltd, Bristol, U.K.) \\ Ben Waterson and Mike McDonald \\ (Transportation Research Group, University of Southampton, U.K.)
}

\begin{abstract}
This paper reports on results of a study undertaken in the U.K. aimed at investigating factors affecting the car following process. An understanding of the factors affecting this complex decision making process is essential to a wide range of theoretical issues including driver workload, capacity and the modeling of freeway flow, as well as practical applications such as the design of in-vehicle driver aids and assistance systems, many of which have direct relevance to increasing driver safety. The study used an instrumented vehicle to collect time dependent following data for a group of test drivers. Data was collected on two differing types of high speed road, using six primary subjects who drove a test vehicle, supplemented by data on one hundred and twenty three drivers that were observed following the test vehicle. Examination was made of how the time headway chosen by a driver is influenced by a range of situational variables commonly believed to effect behavior, with four main findings. Firstly, headway was found to change according to the type of vehicle being followed (i.e. subjects followed closer to trucks than to cars), secondly, little variation was found with changes in overall traffic flow, thirdly, little correlation was found with road type, and lastly a distinct day-to-day variation in individual behavior was observed.

*Contact address
\end{abstract}

Egis Mobilite UK Ltd

Park House

10, Park Street

Bristol

BS1 5HX

U.K.

Email: mark.brackstone@egis-mobilite.co.uk

Key Words:

Driver Behavior, Following Headway, Instrumented Vehicle 


\section{INTRODUCTION}

Despite the recent surge in both simulation modeling and Intelligent Transport Systems (ITS) for motorway and vehicle applications, a lack of detailed understanding of how traffic conditions affect driver behavior still exists. For example, the role of human (mis-) perception and differences between individuals (Brackstone, 2000, Taieb-Maimon and Shinar, 2001) is only now being recognized as a vital contributing factor to the understanding of the design and operation of many in-vehicle systems (Dingus et al., 1997, Marsden et al., 2001), as well as being a causative mechanism in traffic processes such as flow breakdown (Treiber, et al., 2005). The research described in this paper is a detailed evaluation of one particular aspect of behavior, namely the choice of following headway behind the vehicle in-front, examining how this choice, part of the 'car-following process' (Brackstone and McDonald, 2000), varies with local conditions and assessing if there is any evidence of this choice being affected by the behavior of motorists one or more vehicles ahead.

Many studies (from for example Chandler et al., 1958, through Leutzbach and Wiedemann, 1986, to Allen et al, 1997) have developed and supported the concepts and theories of car following. However, almost all have been limited to consideration of the relationships between immediately following vehicles. In many cases existing analyses have either not considered or not controlled for potentially contributing independent variables, or have been carried out in restricted simulated environments where only a simplistic representation of traffic can be created. The development of the instrumented vehicle technique (Brackstone et al., 1999) however means that microscopic field data can now be collected in situ, and in the study described in this paper, innovative on-road experiments have been conducted to examine in detail four hypotheses that may play an important role in the ability to understand and model vehicle following.

i) The level of traffic flow affects driver behavior. Here, it is suspected that increases in flow and density may lead the driver to expect an increasing probability (and severity) of unpredictable braking events with a consequential increase in following headway due to safety concerns, or alternatively a reduction in headway as drivers seek to prevent vehicles 'cutting in' in front of them.

ii) Driver following behavior varies with road characteristics. There are many reasons to believe that road type itself may have an affect on following behavior, for example, the frequent stop and start nature of traffic occurring on urban roads, interactions with merging vehicles, traffic control systems such as traffic signals, the presence of pedestrians and a wider range of road users may necessitate a higher degree of preparedness. This in-turn could result in increased headways on urban roads than would occur under equivalent conditions on interurban roads.

iii) Driver following behavior is affected by the type of lead vehicle. Anecdotal opinion suggests that drivers should follow trucks/vans at greater headways than cars, as trucks obscure more of the forward view than cars and hence reduce drivers' warnings of, and time to adjust to, sudden changes in traffic speed. However, the 
very few studies that have investigated this issue have found the inverse, with trucks being followed closer than cars (Parker, 1996, Sayer et al., 2003).

Drivers are inconsistent in their choice of headway. Minor changes in for example motivation, aggression or alertness may unknowingly influence the headway adopted by a driver and may in effect, act as a 'background noise' or variability, hampering the ability to provide a predictive model for any one driver or group of drivers. No previous attempts have been made to establish the magnitude of such effects, which could mask, or at worst be taken as a surrogate for, the effect of other variables.

Both a lack of understanding of the behavioral issues involved in these hypotheses and a lack of sufficient data have been major reasons why clear cut findings have not previously been available. The study reported in this paper therefore attempts to address these hypotheses by measuring following headway in as wide a variety of conditions as possible on real roads, and to separate out variables that have up until now been confounded in analyses.

Firstly a brief overview of the following processes which form the core of this research is presented, before the data collection methodology and resulting database are described. Results from extensive analyses of variance are then detailed, accompanied by an interpretation of these results in relation to the four hypotheses and an assessment of their significance in the context of other recent studies. In the final section, the conclusions provide an overview of the impact of this study on future data collection exercises and, in particular, all studies reliant on driver following relationships.

\section{FOLLOWING PROCESSES}

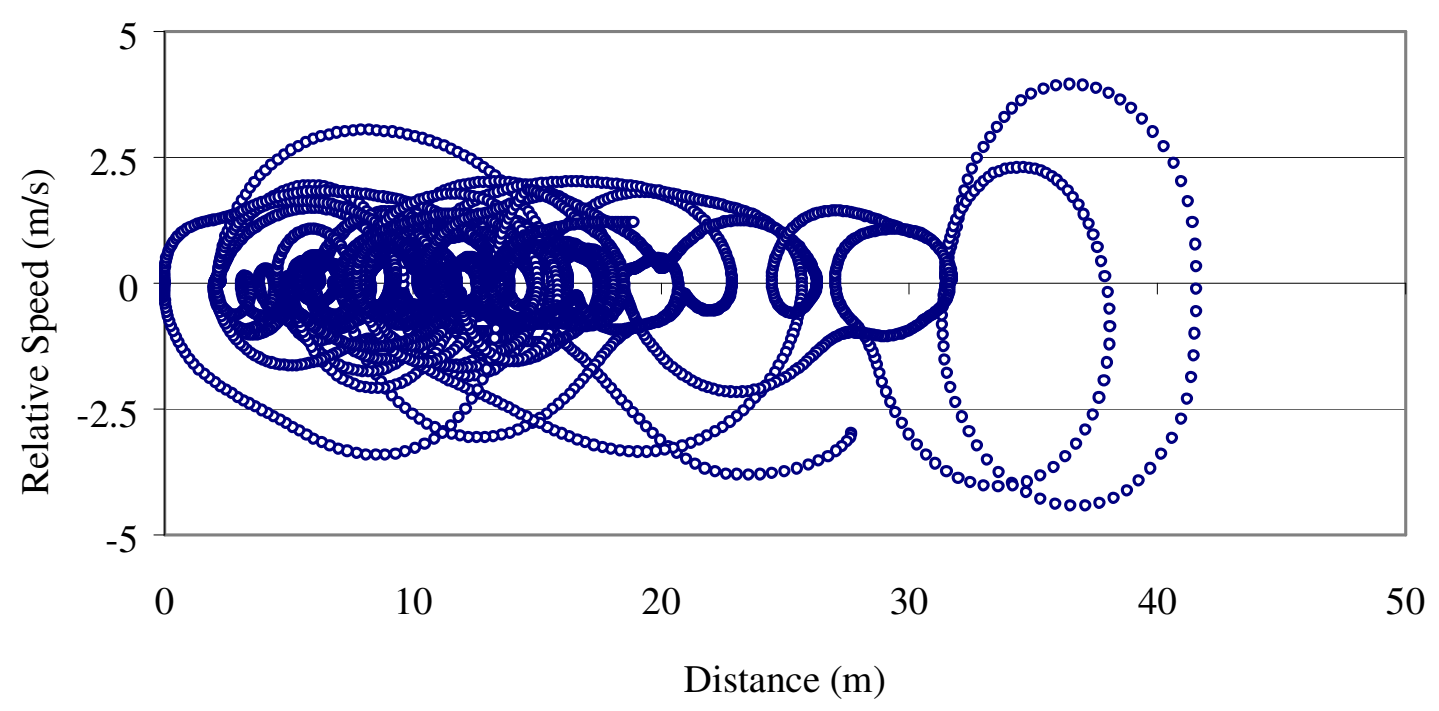

Figure 1: A typical following relationship showing variation of relative speed and distance.

The basic assumption in car-following (or more generally, vehicle-following) is that each driver attempts to maintain a desired following headway behind the vehicle in-front, (the lead vehicle) through modulation of the throttle and brake. This continuous adjustment process is influenced by driver perceptions of ground speed, proximity to their desired headway, and the 
dynamics of the relationship with the preceding vehicle (Brackstone and McDonald, 2000), typically represented in models by the relative speed (DV) between the two vehicles (negative closing), the angular velocity of the lead vehicle ('looming', $\Theta_{\mathrm{t}}$ ) or the Time to Collision. (TTC $=$ rear to front intervehicle spacing (DX) divided by the relative speed $=$ DX/DV). Such continuous adjustments of the vehicle speed over time according to these behavioral responses typically then leads to following 'spirals' (Figure 1) which can be expressed in the DX-DV plane.

Such spirals have clear implications for both the safety of the individual driver, the flow/capacity of the road as a whole, and the collective safety of all drivers, particularly at times of high demand where following is common. Although such processes can occur at any speed, at low demand (as illustrated in Figure 2) drivers are mostly able to drive at their desired speeds, and those with higher desired speeds have ample opportunity to pass slower vehicles. As demand increases however, the frequency of following increases as does its importance, as passing opportunities become less common, leading to the building of platoons of vehicles. Eventually, disturbances within these platoons due to natural speed variations (viz. following spirals) increase to such a level that the speed of those involved is reduced, increasing the differential between desired and attainable speeds still further. Flow may thus become unstable with shock waves and flow breakdowns leading to increased accident risk and reduced journey time reliability. Driver behavior near these critical points has long been an area of interest as minor changes in individual and/or collective behavior of drivers may effect the flow levels at which flow breakdown occurs and the severity of the breakdown. Potentially drivers may exhibit some manner of anticipational behavior through observation of the behavior of the traffic flow surrounding them.

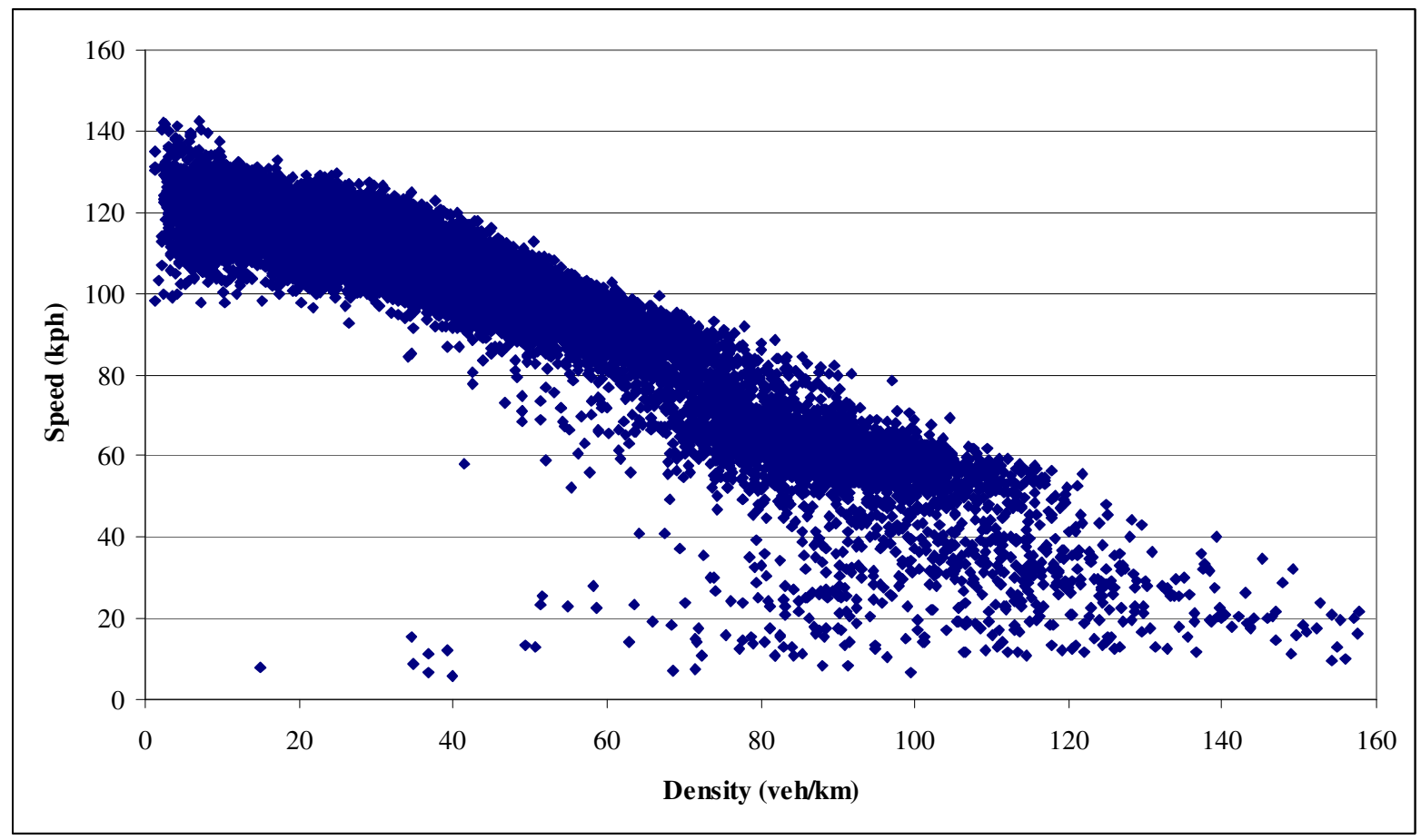

Figure 2: The inter-relationship of speed and density. 


\section{DATA COLLECTION}

Data used for this study was collected using the TRG Instrumented Vehicle (Brackstone et al., 1999), a 2 litre saloon car equipped with sensors capable of simultaneously measuring: ground speed; distance to adjacent vehicles (through automotive radars), all at $10 \mathrm{~Hz}$; and recording video pictures of driver, rear, and forward views.

Two test routes were used for data collection, one a three lane motorway (70mph $\sim 113 \mathrm{kph}$ speed limit), the other an urban arterial two/three lane carriageway (50 $\mathrm{mph} \sim 80 \mathrm{kph}$ speed limit). For the motorway test route a $16.75 \mathrm{~km}$ unidirectional course was chosen (the M27 to the north of the city of Southampton in the U.K., running east to west between Junctions 5 and 1) which was heavily congested during the evening peak (17:00 to 18:00). The urban test route consisted of $4.5 \mathrm{~km}$ of the A35 running from the centre of the city of Southampton to its western suburbs, with data collection being undertaken in the congested (westbound) direction, during the evening peak (17:00-18:00). The A35 course consisted of a 2 lane through-section, with an additional (third) lane being gained and lost at two consecutive atgrade intersections and with a signal controlled four-way intersection occurring approximately $1 \mathrm{~km}$ along the test course. (It should be noted that following behavior may vary when in immediate proximity to traffic signals, with the driver having to consider whether to 'stop' or 'go' should the signals begin to change from green to red. However such a consideration will only be likely to effect behavior for a small period of time, while the driver is close to the junction, otherwise the behavior will probably be identical to that at low speed caused by other effects such as queuing. Therefore, for the purposes of this analysis such effects have been discounted).

Data was collected for two groups of drivers. The first were 'active' participants, six subjects recruited to participate and given one-off payments (aged between 29 and 54, average age 41). All were experienced in both driving the test vehicle and driving on the roads used in this research, minimizing any potential learning effects. Data collected for these subjects involved measuring the following distance from a front facing radar to the rear of the vehicle in-front. The second group were 'passive' subjects who were observed following to the rear of the instrumented vehicle during data collection runs using the 'active' subjects. Data collection on these subjects involved measuring the distance to those vehicles following the instrumented vehicle.

Collection of data on 'passive' subjects supplements the 'active' database by allowing for a greater number of drivers to be sampled. However, no control could be exercised over the subject observed and, for example, no information on personal characteristics are available and the total duration of observation is dependant on how long the follower wished to remain behind the test vehicle. Typically this resulted in one time series over a small range of speeds for each observed follower. (As the instrumented vehicle is equipped with two rangefinders, measurement of both lead and rear vehicles was possible in some instances, enabling the simultaneous dynamics of three consecutive vehicles to be measured. However, for the purposes of this paper, only two-vehicle dynamics are examined, based on data from both active and passive collection techniques available for each route). 
For the 'active' subjects, each test driver attempted five test drives, each on separate days, three on the motorway route, and two on the urban route, being instructed to drive the vehicle as they would normally for all data collection runs. The first of the motorway drives was a 'high speed' run between 12:00 and 13:00 when traffic flow was low, with the driver allowed to drive freely. Subsequently, two runs were made during peak periods, in one of which the driver was instructed to follow an identified lead vehicle (an easily visible white van which obscured the view of the traffic ahead) and in the other with the driver allowed to choose any car to follow. On the urban route, each driver attempted two runs at peak times, being allowed to choose their own vehicle to follow, however due to equipment failure data was only available for one drive for subject 5 .

All data was collected in dry conditions in the winter of 2000/01 for the motorway course, and the summer of 2001 on the urban course. Interference with visibility due to low sunlight is not believed to have affected driver behavior due to the precise orientation of the courses. A total of 501 vehicle-following time series were collected for active and passive drivers, with each consisting of uninterrupted streams of information on the following behavior of a single vehicle, from initial acquisition of a lead vehicle to the termination of the following process. Initial acquisition was defined as being when the rear vehicle either closed to less than a two second headway (when car following is commonly considered to commence, Reiter, 1994), or assumed a 'stable' following distance (a relative speed of less than $1 \mathrm{~ms}^{-1}$ ). Termination of the process was defined as either a lane change or an increase in headway to more than two seconds. The number of sequences collected using each technique and route, the average speed of the vehicles observed, and the average of the maxima and minima vehicle decelerations observed in each series, are given in Table 1.

\begin{tabular}{l|ccccc} 
& $\begin{array}{c}\text { Number of } \\
\text { Sequences }\end{array}$ & $\begin{array}{c}\text { Ave. Length } \\
(\text { sec. })\end{array}$ & $\begin{array}{c}\text { Ave. Speed } \\
(\mathrm{kph})\end{array}$ & $\begin{array}{c}\text { Ave. Max. } \\
\text { Decel. }\left(\mathrm{ms}^{-2}\right)\end{array}$ & $\begin{array}{c}\text { Ave. Max. } \\
\text { Accel. }\left(\mathrm{ms}^{-2}\right)\end{array}$ \\
\hline Motorway Active & 237 & 52 & 96.4 & -0.8 & 0.7 \\
Motorway Passive & 62 & 102 & 92.0 & -1.1 & 1.1 \\
Urban Active & 139 & 102 & 29.4 & -1.9 & 1.6 \\
Urban Passive & 63 & 224 & 33.5 & -2.1 & 2.0
\end{tabular}

Table 1: Summary statistics of data collection.

\section{Variables Considered}

Seven variables have been used in this study:

a) Three variables defining or derived from the vehicle dynamic state. These include the response variable time headway between vehicles ( $\mathrm{TH}$ sec.), calculated as the following distance divided by the speed of the following vehicle. Distance was measured from the rear of the preceding vehicle to the front of the instrumented vehicle in the active case, and the front of the following vehicle to the rear of the instrumented vehicle in the passive case. Two potential explanatory variables, the ground speed of the following vehicle $\left(\mathrm{V} \mathrm{ms}^{-1}\right)$ and the relative speed between the two vehicles (DV $\mathrm{ms}^{-1}$, with negative values representing a higher speed for the following vehicle). (Time Headway was chosen as the primary indicator of driver 
performance in this investigation. Although Time To Collision (TTC=TH.V/DV) could alternatively have been used, this paper focuses on stable following behaviour, defined in more detail below, which, by virtue of the low values of DV, makes accurate assessment of TTC difficult.).

b) Four situational variables have been used as explanatory variables, including subject (SUB), type of leading vehicle (VEH, car or truck/van), road type (ROAD, M27 or A35) and flow (FLOW, vehicles/hour $\left(\mathrm{vh}^{-1}\right)$ ). FLOW was calculated based on information measured by three induction loops located on each of the two test routes. Because of the changing nature of flows, each flow measurement was taken to be valid only for thirty seconds before and after the passage of the test vehicle. Although approximate (and leading to flow data not being available for all points within the dataset), no other measurement options were available, with geometry and lack of suitable vantage points precluding video monitoring of the majority of the test routes.

In order to better investigate the dependencies and the non-linearities in the data, ground speed (V) and flow (FLOW) were converted from covariates into factor variables. Speed category was classified in multiples of $5 \mathrm{~ms}^{-1}$ (SPEEDCAT, seven levels up to $35 \mathrm{~ms}^{-1}$ ) and flow category was classified in multiples of $1000 \mathrm{vh}^{-1}$ (FLOWCAT, six levels up to $7000 \mathrm{vh}^{-1}$ ) respectively.

\section{Following Headway}

An inspection of the time series for each following 'sequence' immediately shows that there is a great deal of variation in both relative distance and speed between lead and following vehicle. This can best be displayed as a 'following spiral' such as the one shown in Figure 1 where DX is plotted against DV for such a following sequence. Although not the focus of this paper (the reader is referred to Brackstone et al., 2002, for further details) it is important to understand the presence of this oscillatory process before analysis can start. For example, it is clear that higher DV values do not represent the following distance or headway desired by the driver, but rather describe regimes where the driver is drifting to or from a desired headway. In order to remove such points the data analyzed has first been filtered to remove points where the magnitude of the current acceleration exceeds $\pm 0.6 \mathrm{~ms}^{-2}$, thus producing 'stable' following points. This threshold has been selected from previous studies as representing a minimum acceleration level above which drivers are typically adjusting their speed (Sultan, 2000). Thus it is most likely that when below this threshold the driver is at (or close to) the following distance that they most desire. Subsequent filtering of following data included a reduction in the database, sampling one point in every ten consecutive readings to reduce the effects of autocorrelation.

Such experiments in real conditions are perforce restricted by a lack of control, with it proving exceptionally difficult to ensure that all variables and factors are represented equally in the design. The quantity of data collected for this study however still represents the highly diffuse nature of following behavior, accompanied by corresponding explanatory information. 


\section{DATA ANALYSIS AND RESULTS}

\section{Selection of Data and Approach}

Before analysis can be undertaken it is important to consider a key limitation of the passive dataset. Many of the passive subjects would, due to the comparatively short period of observation, not produce following data with a wide variety of speeds, and due to the short spatial distance over which they were observed such data would not necessarily be able to be correlated with the flow readings taken at specific sites. As a result, data collected from the six drivers recruited specially for the 'active' phase was used to formulate the model, which was then validated on the larger 'passive' dataset.

\section{Analysis of Active Data}

Initial analysis of data from the six principle subjects revealed that there is a limited dependence of time headway (TH) on speed (V) alone. For example, attempts to fit any of the common relationships (linear, quadratic, exponential, etc.) to the data met with limited success, with a linear fit yielding an $\mathrm{R}^{2}$ (goodness to fit) value of only 0.274 , while the most successful found was an inverse relationship with $\mathrm{TH}=1.03+4.31 \mathrm{~V}^{-1}$, with an $\mathrm{R}^{2}$ value of 0.363. In particular, it was clear there that there were differences between subjects with the average value of $\mathrm{TH}$ for each subject varying between 0.80 and $1.71 \mathrm{sec}$. for the M27, and between 1.06 and $2.54 \mathrm{sec}$. on the A35 where speeds were slower and flows were higher (Figure 3).

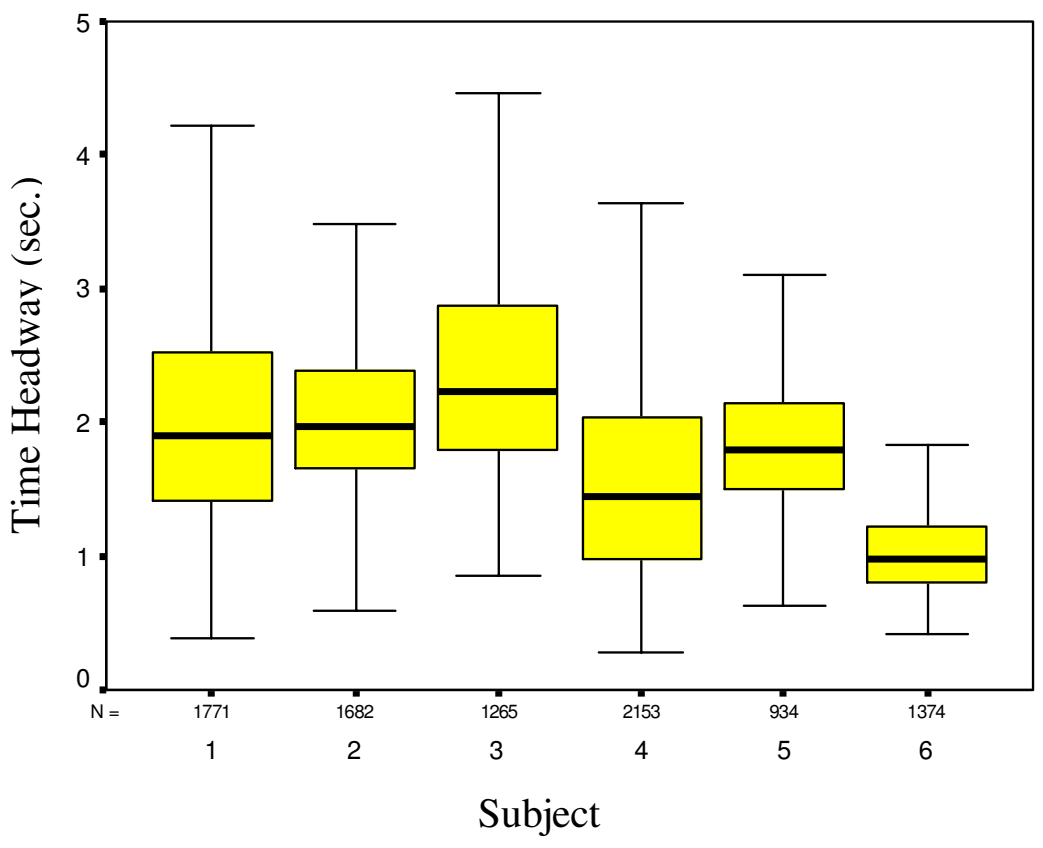

Figure 3: Following distances exhibited by each driver during A35 active data collection.

Univariate analysis of variance techniques were therefore used to examine the data. An initial model using, SUB, SPEEDCAT, VEH, FLOWCAT and ROAD was investigated based on the 6276 active data points available which contained associated FLOW datum (approximately 
$30 \%$ of the total of 21,100 active data points). It must be noted that with the large volume and range of data points collected in this study, finding significant relationships within the data is not in itself difficult, however the key issue is finding those relationships which most help in producing meaningful predictions of time headway. The analysis was therefore restricted to main effects and second level interactions, each of which are then potentially allowed to vary by subject. Terms (including interactions) were added sequentially to the model according to which variable produces the highest increase in overall goodness of fit, thereby producing as parsimonious a model as possible while ensuring that the structure is able to represent the wide range of behavioral considerations and variabilities.

\begin{tabular}{|c|c|}
\hline Accepted Variable & Other Variables \\
\hline SPEEDCAT $(0.315)$ & $\begin{array}{l}\text { SUB (0.270), ROAD (0.213), VEH (0.105), FLOWCAT } \\
(0.061)\end{array}$ \\
\hline SUB $(0.560)$ & FLOWCAT (0.349), VEH (0.338), ROAD (0.317) \\
\hline SPEEDCAT*SUB $(0.625)$ & VEH (0.576), FLOWCAT (0.568), ROAD (0.560) \\
\hline VEH (0.639) & FLOWCAT (0.632), ROAD (0.625) \\
\hline VEH*SUB (0.652) & $\begin{array}{l}\text { SPEEDCAT*VEH (0.651), FLOWCAT (0.644), ROAD } \\
(0.639)\end{array}$ \\
\hline SPEEDCAT*VEH $(0.660)$ & FLOWCAT (0.658), ROAD (0.653) \\
\hline SPEEDCAT *VEH*SUB $(0.684)$ & FLOWCAT (0.665), ROAD (0.660), \\
\hline FLOWCAT $(0.688)$ & $R O A D(0.684)$ \\
\hline SPEEDCAT*FLOWCAT $(0.729)$ & $\begin{array}{l}\text { FLOWCAT*SUB }(0.710), \text { VEH*FLOWCAT }(0.701), \\
R O A D(0.688)\end{array}$ \\
\hline SPEEDCAT*FLOWCAT*SUB $(0.772)$ & $\begin{array}{l}\text { FLOWCAT*SUB }(0.748), \text { VEH*FLOWCAT }(0.737) \text {, } \\
\text { ROAD }(0.702)\end{array}$ \\
\hline VEH*FLOWCAT (0.774) & ROAD (0.772), FLOWCAT*SUB (0.772) \\
\hline VEH*FLOWCAT*SUB $(0.782)$ & ROAD (0.774), FLOWCAT*SUB (0.774) \\
\hline FLOWCAT*SUB $(0.782)$ & $\operatorname{ROAD}(0.782)$ \\
\hline ROAD (0.782) & \\
\hline
\end{tabular}

Table 2: $\mathrm{R}^{2}$ associated with each model in Time Headway analysis - reduced active database.

The goodness of fit achieved at each stage of the analysis is given in Table 2 (where variables not reaching a $1 \%$ significance level are italicized). It can be seen that two variables (SUB, and SPEEDCAT) and their interaction, alone produced an $\mathrm{R}^{2}$ of 0.625 (compared to a five variable full factorial model $\mathrm{R}^{2}$ of 0.791 ), with the subsequent addition of VEH and its associated interaction terms produced a further significant increase to model fit with $\mathrm{R}^{2}$ increasing to 0.684 (an increase to $86 \%$ of the full factorial $\mathrm{R}^{2}$ ). The predictive accuracy of this model is then only marginally improved by the addition of FLOWCAT or ROAD and their associated interaction terms, suggesting that these variables (particularly ROAD) may be taken to have minimal effect on observed behavior. A re-application of this model considering only SUB, 
SPEEDCAT and VEH related terms to the entire database (i.e. additionally including those records not containing corresponding flow measurements) yields only a small reduction in $\mathrm{R}^{2}$ (from 0.684 to 0.513 , Table 3 ), emphasizing the robustness of the model formulation .

\begin{tabular}{l|l} 
Accepted Variable & Other Variables \\
\hline SPEEDCAT (0.299) & ROAD $(0.164)$, SUB $(0.152)$, VEH $(0.091)$, \\
SUB $(0.426)$ & VEH $(0.303)$, ROAD $(0.299)$ \\
SPEEDCAT*SUB $(0.493)$ & VEH $(0.430)$, ROAD $(0.427)$ \\
VEH $(0.500)$ & READ $(0.494)$ \\
SPEEDCAT*VEH $(0.505)$ & VEH*SUB $(0.504)$, ROAD $(0.500)$, \\
SPEEDCAT*VEH*SUB $(0.512)$ & ROAD $(0.512)$ \\
VEH*SUB $(0.513)$ &
\end{tabular}

Table 3: $\mathrm{R}^{2}$ associated with each model in Time Headway analysis - full active database.

\section{Comparison of Model with Passive Database}

In order to examine if the model formulated for the six 'active' subjects is broadly representative of the driving public as a whole, the formulated model was re-applied to data from the remaining ('passive') subjects for which data had been collected. A full examination of the model applicability is hampered due to the restricted ranges of speed that many of the subjects were observed over (small variations in speed essentially lead to SPEEDCAT becoming analogous to SUB), and some degree of selection has to be made of the validation data to be used. Therefore, for the purposes of this comparison, only subjects that were observed as contributing data to four or more of the seven speed categories, and contributing a minimum of 100 data points in total were selected. When applied to the data from the thirty five 'passive' subjects found to fit these criteria, the 'active' model was found to fit the 'passive' data with an $\mathrm{R}^{2}$ of 0.632 . Comparison of the unstandardised residuals of the model applied to the six active subjects and the additional set of thirty five passive subjects was undertaken to ensure that the active sample was representative of the population as a whole. While both sets of residuals were normally distributed about zero, an $\mathrm{F}$ test revealed a significant difference in variance between the two sets $\left(\mathrm{F}_{25070,10236}=2.143, \mathrm{p}<0.001\right)$. Therefore it seems that although the responses of the six subjects used were otherwise a representative sample of the driving population as a whole, a much larger degree of variation was present in their behavior, although this is to be expected considering the large difference in the quantity of data collected per driver for the two groups of subjects. 


\section{Interpretation}

Before interpreting the models produced by this analysis it is important to briefly assess the validity of using the univariant analysis of variance approach. While exploratory examination of the residuals from the model support the assumption of normality and constant variance within the error structure, issues remain over two of the model assumptions. The first concerns the independence between the data points, specifically those occurring within the same following series. If this analysis was based on all the available data then high correlations between successive response measurements and explanatory variables (such as in Figure 1) would lead to, at best, large underestimating of the residual mean square error and at worst, significant bias within the parameter estimates. However, as the data used for the model was sampled from this recorded data set at one second intervals, these effects should be reduced to negligible levels and there should be little bias in the parameter estimates. The second assumption is that of causality. While not requiring a causal relationship between the explanatory and response variables, the analysis would be significantly affected if there was a reverse relationship (for example if the time headway determined which subject was driving). Clearly for subject, road and (due to its macroscopic nature) flow this does not happen, but the existence of car-following 'spirals' suggests that speed could be considered a function of time headway. This affect was removed as the analysis described here considers only stable following events where changes in speed are small, and broader speed categories rather than individual speeds.

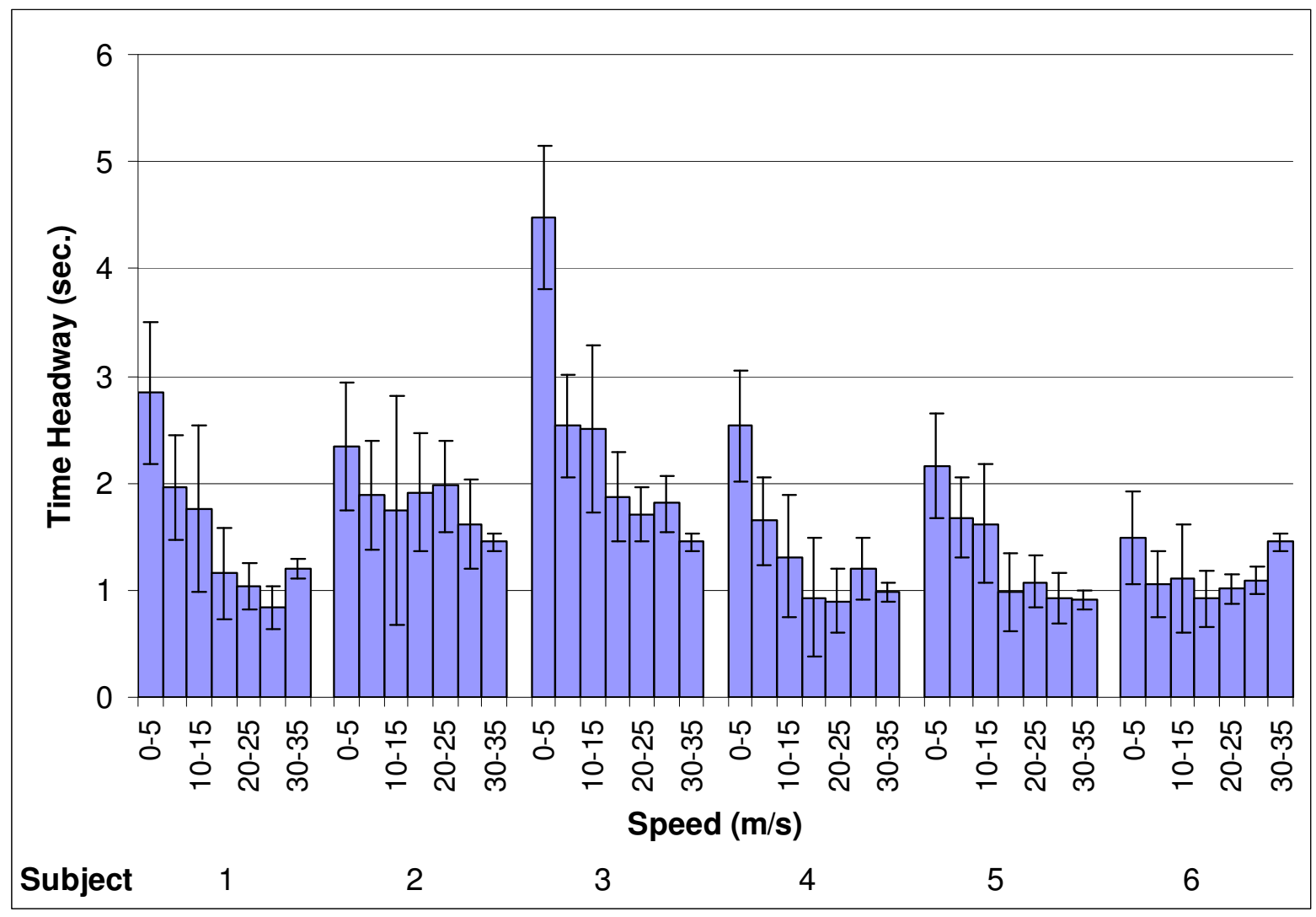

Figure 4: Variation of predicted (car) following headway by subject and speed. 
An examination of the model parameters and corresponding predicted following headways based on the three explanatory variables (SPEEDCAT, SUB and VEH and their interactions) reveals a large degree of variation between subjects (Figure 4), with time headway being far from constant either between or within subjects. However, while the degree of between subject variation makes it difficult to discern any clear overall trend, averaging across subjects (Figure 5) provides an illustration of the underlying pattern. At this level it is possible to split driver response into two regimes. Firstly, an area of approximately constant time headway above $15 \mathrm{~ms}^{-1}$, and secondly an area of increasing time headway below that threshold.

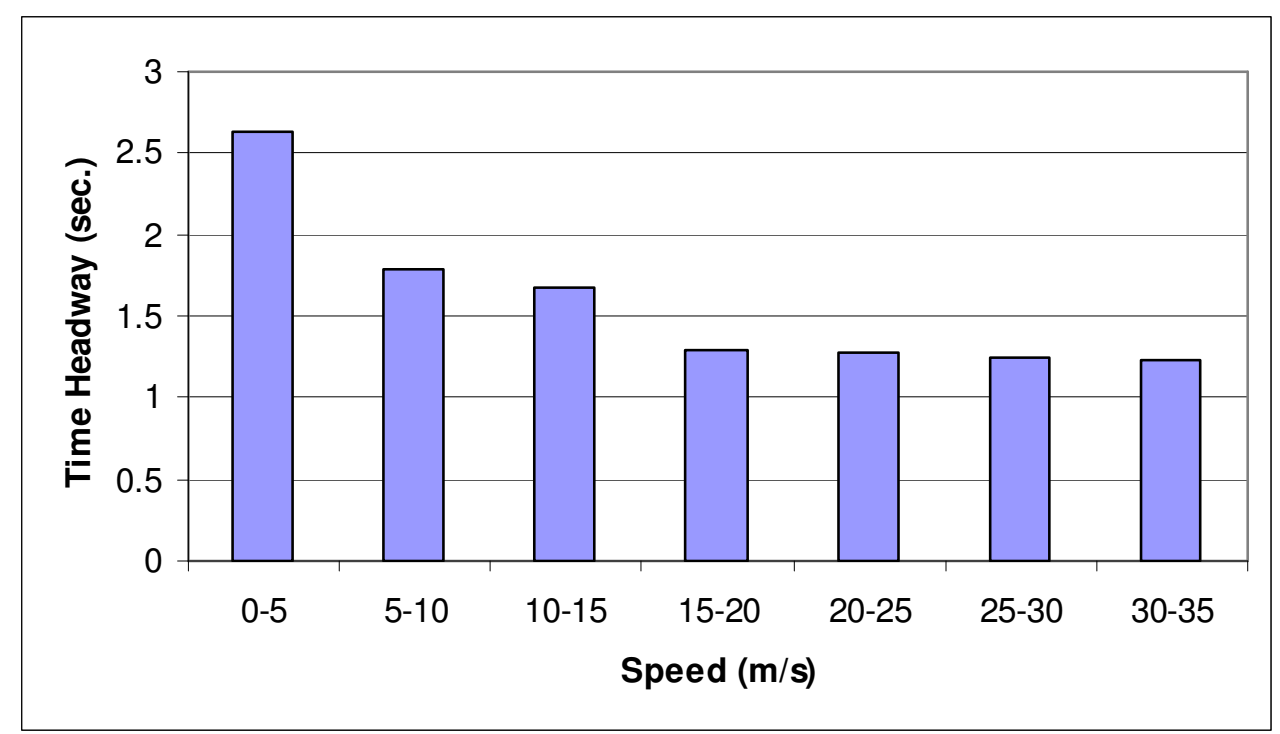

Figure 5: Variation of predicted (car) following headway by speed.

It has been hypothesized that following headway may be affected by the type of vehicle being followed and, as has been demonstrated, the nett effect of the VEH variable is far less than that of either SUB or SPEEDCAT although some effect is present. Quantifying the precise effect from the dataset however is not straightforward, as in many cases (Table 4) few or no van following series had been observed in many of the speed categories (only $7.8 \%$ of the truck/van related observations occur below $20 \mathrm{~ms}^{-1}$, and only $3.9 \%$ below $15 \mathrm{~ms}^{-1}$ ).

\begin{tabular}{c|cccccc|c} 
Speed (kph) & Subject 1 & Subject 2 & Subject 3 & Subject 4 & Subject 5 & Subject 6 & All \\
\hline $0-5$ & 43 & & 60 & 9 & & & 112 \\
$5-10$ & 40 & & 80 & 21 & & & 141 \\
$10-15$ & 26 & 4 & 35 & & 7 & & 72 \\
$15-20$ & 36 & 53 & 153 & 14 & 76 & & 332 \\
$20-25$ & 191 & 336 & 988 & 356 & 161 & 454 & 2486 \\
$25-30$ & 808 & 427 & 394 & 720 & 934 & 1001 & 4284 \\
$30-35$ & 586 & & & 57 & 162 & 192 & 997 \\
\hline All & 1730 & 820 & 1710 & 1177 & 1340 & 1647 & 8424
\end{tabular}

Table 4: Van following data points by subject and speed.

This limitation means that a robust examination can only be undertaken of the additional effects that VEH based terms have on following headways above $20 \mathrm{~ms}^{-1}$, where it may be seen (Figure 6 where positive values indicate following closer to trucks than cars) that if 
averaged across speeds there is a net decrease in headway for a car driver following behind a truck (a negative adjustment) of from 0.03 to $0.39 \mathrm{sec}$. Two of the subjects exhibited a small negative mean adjustment (subjects 4 and 5) and the others a clear negative shift of $0.25 \mathrm{sec}$. or more. Also interesting is that in four of the six subjects there is a clear negative trend, with the reduction in time headway being more pronounced at higher speeds.

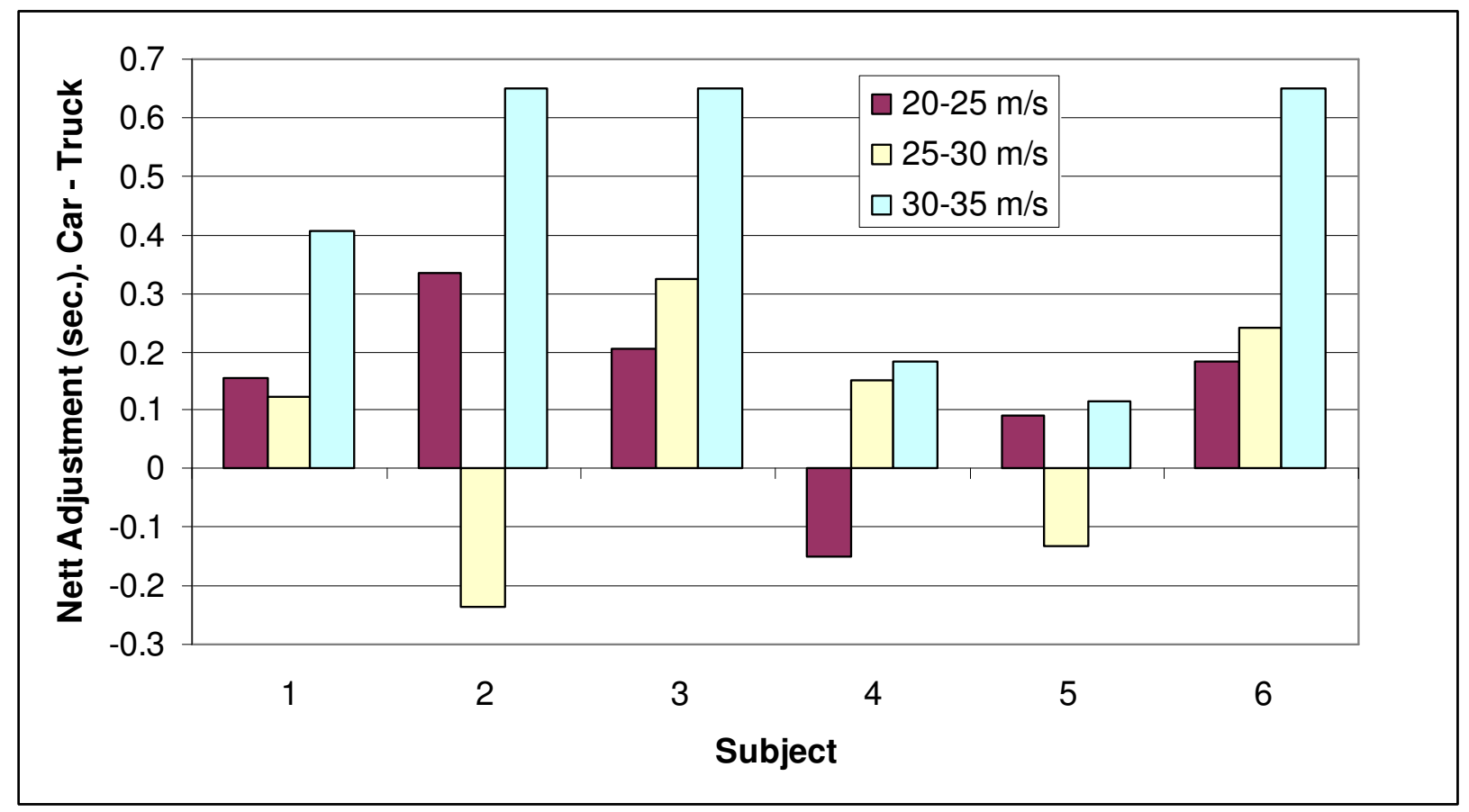

Figure 6: Comparison of car following and truck/van following headways.

\section{Day-to-Day Variability}

With five of the 'active' drivers having undertaken two test drives under nominally identical conditions on the urban course, it is possible to examine how their behavior may have varied while also controlling for the explanatory variables. A further analysis was therefore performed on the predicted values from the model for data from the A35 (urban) test runs. The results of this analysis (shown in Table 5), examined the variation of the residuals (observed data predicted data) according to subject and test drive, finding that significant variation existed between the pairs of distributions of the residuals, demonstrating between day variations occurring at the $1 \%$ level for four of the five subjects. The nett changes in headway for these subjects between the trials ranged from $0.18 \mathrm{sec}$. up to $0.27 \mathrm{sec}$., corresponding to around 5.7 $-8.6 \mathrm{~m}$ at $70 \mathrm{mph}(113 \mathrm{kph})$ and $4.0-6.1 \mathrm{~m}$ at $50 \mathrm{mph}(80 \mathrm{kph})$.

It can be seen that in all cases the mean of the residuals was increased in the second trial for these four subjects (with a decrease in the standard deviations in three of the cases), clearly indicating a day-to-day difference. Although it is possible to hypothesize that this could be a learning process, with drivers settling in and over time exhibiting headways more reflective of their 'usual' behaviour, there are several facts that should be considered. Firstly, by the time the Urban runs were being performed each subject had already driven the car three times, hence it is unlikely that there would be any extra familiarity to be gained with the 
characteristics of the vehicle and, even if there were, the separation between runs (a matter of weeks) in this case would most likely have reduced any working memory of the handling characteristics. Secondly all of the subjects were familiar with the route having driven it 'regularly' over several years as part of their weekly driving patterns. Regardless of whether some manner of systematic shift is occurring here or not it is abundantly clear that significant variations can and do occur between different trials that cannot be explained by the variables considered. Although doubt may be cast over whether this effect would occur in drivers using their own vehicles, it is clear that it does occur in field experiments where the driver is under observation, and this has clear and important implications as to how such trials can and should be conducted in the future.

\begin{tabular}{|c|c|c|c|c|c|c|c|c|c|c|}
\hline \multirow[t]{2}{*}{ Subject No. } & \multicolumn{2}{|c|}{1} & \multicolumn{2}{|c|}{2} & \multicolumn{2}{|c|}{3} & \multicolumn{2}{|c|}{4} & \multicolumn{2}{|c|}{6} \\
\hline & $\sum^{\mathbb{E}}$ & $\frac{\dot{\vec{\nu}}}{\stackrel{0}{0}}$ & 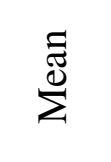 & $\begin{array}{l}\dot{\vec{\nu}} \\
\dot{\overrightarrow{0}}\end{array}$ & $\sum_{\Sigma}^{\tilde{\Xi}}$ & $\frac{\dot{d}}{\stackrel{D}{0}}$ & $\sum_{\Sigma}^{\Xi}$ & $\begin{array}{l}\dot{\overrightarrow{0}} \\
\frac{\overrightarrow{0}}{i s}\end{array}$ & $\sum_{\Sigma}^{\tilde{E}}$ & $\frac{\dot{0}}{\overrightarrow{0}}$ \\
\hline Test Run 1 & -0.09 & 0.76 & -0.07 & 0.64 & -0.11 & 2.15 & -0.11 & 0.58 & 0.01 & 0.31 \\
\hline Test Run 2 & 0.07 & 0.88 & 0.11 & 0.56 & 0.16 & 0.93 & 0.11 & 0.47 & -0.01 & 0.40 \\
\hline $\begin{array}{l}\text { F value of } \\
\text { difference in } \\
\text { means }\end{array}$ & $\mathbf{F}(\mathbf{1}$, & $\begin{array}{l}769) \\
6.41\end{array}$ & $\mathbf{F}(\mathbf{1}$, & $\begin{array}{l}680) \\
6.34\end{array}$ & & $\begin{array}{l}1263) \\
=6.98\end{array}$ & & $\begin{array}{l}1978) \\
79.62\end{array}$ & & $\begin{array}{l}1372) \\
=1.25\end{array}$ \\
\hline
\end{tabular}

Table 5: Distribution of Residuals (sec.) for Time Headway Model according to subject and Trial. Bold indicates significant differences in the means at the $1 \%$ level. (Note, no comparison possible for Subject 5 due to insufficient data).

\section{DISCUSSION}

In the analysis presented above an examination has been undertaken of the impact that a number of situational factors may have on the choice of 'following headway' and it has been shown that such factors introduce significant variation in what has often been assumed to be a simple, highly predictable, variable. Independent verification of the results obtained in this research is not straightforward however, as relationships in existing published work have been compiled over some time (during which driving behavior may have changed), in differing countries (where differing driving codes and enforcement processes may affect results), under differing conditions (e.g. at road works) or using different methods of data collection (e.g. simulators). The implications of this problem are evident when considering each of the original hypotheses of this study:

\section{i) The level of traffic flow affects driver behavior.}

Rejected: Little evidence was found within this research to support this hypothesis

Surprisingly, the full impact of flow on following behavior is an issue that has rarely been examined prior to this study. Aron, Biecheler, Hakkert and Peytavin (1997) for example examined car following in differing flow conditions, although no attempt was made to control for speed. Dijker, Bovy and Vermijs (1998) compared measurements based on several hours induction loop data for 'congested' and 'non congested' conditions (where average speeds 
were observed as below or above capacity speed respectively), finding differences in following distance either side of a critical point of around seven meters. However, being based on many single observations of different drivers, Dijker's study gives us little indication of the how such an effect is distributed over individuals, or indeed what differences may occur due to differing densities at comparable speeds. A more microscopic analysis has been provided by TaiebMaimon and Shinar (2001) (an on-road experiment using 30 volunteers) examining the differences between a driver being given a 'comfort headway' and a 'minimum headway' driving instruction. (Which could be taken as a surrogate of 'choice' and 'forced' following), giving a difference of 5-10m. However, in such an experiment the driver would have no reason to believe that the speed of the lead vehicle would be about to change, as would really occur in high flow conditions.

Perhaps the most interesting finding however was in a simulator experiment undertaken by van der Hulst, Meijman and Rothengatter (1998), where changes in reaction time demonstrated that if a driver is aware that a vehicle several cars in-front is decelerating or about to decelerate, then they may deliberately start to increase their headway in order to minimize any likely future incursion within a minimum headway safety zone. Although logical, this finding must again be treated with caution due to the established limitations of simulators in presenting the driver with a realistic picture of a congested road (resolution and processing limits restricting the number of vehicles that may be shown to the driver at any instant). The finding presented here, showing that flow has little or no effect on headway, is not totally at odds with what has been found elsewhere as varying flow itself has not strictly been investigated by other authors, who instead have presented results that demonstrate more the effect of anticipation or intent, rather than the complex combination of the two which may be generated in practice once a driver is faced with a large number of closely spaced surrounding vehicles.

\section{ii) Driver following behavior varies with road characteristics.}

Rejected: No effect of road type was found within this research.

Although most observations of following behavior have been made on motorways, some studies have been made relating to behavior on other types of roads (for example Rajalin et al., 1997, Michael et al., 2000). However, the results are of little relevance as this study has demonstrated the importance of between subject variability, and comparison of behavior should be made for 'identical' populations and not between experimental groups. One of the few indications that may exist as to qualitative differences comes from Conchillo, Nunes, Recarte and Ruiz (1999) who, in measuring subjectively perceived speed, demonstrated that the differences between perceived and actual speed for a highway and urban road at the same speed levels could be of the order of up to $8 \mathrm{kph}$. Although this does not translate to a directly observable behavioral effect it would lead one to believe that on roads with lower speed limits/more difficult geometry, drivers operate with an increasing difference between perceived speeds and actual speeds. One may argue that this difference could result in differing headways however our results do not seem to bear this out with there being no significant difference found in distance keeping behaviour between the road types. Even if there may be a difference in speed perception it would seem therefore that choice of following distance does not seem to be linked it and other associated variables such as the complexity of, and the rate of change of, the visual field for example. 
This is a post-peer-review, pre-copyedit version of an article published in Transportation Research Part $F$ : Traffic Psychology and Behavior. The definitive publisher-authenticated version-Brackstone $M$, Waterson B \& McDonald M (2009) "Determinants of Following Headway in Congested Traffic", Transportation Research Part F: Traffic Psychology and Behavior, 12(2), p131-142 - is available online at: http://dx.doi.org/10.1016/j.trf.2008.09.003

iii) Driver following behavior is effected by the type of lead vehicle.

Confirmed: Evidence was found supporting this hypothesis with (in general) drivers being found to follow closer behind trucks/vans than cars.

Direct statistics on comparable behavior are hard to acquire, for example the often cited study by Postans and Wilson (1983) quotes frequencies of 'tailgating' (headways less than half a second in this case) and how these vary by vehicle type, but does not actual quantify the magnitude of this tailgating. Similarly, Sumner and Baguley (1978), while measuring differences in how car and truck drivers follow lead vehicles, present no results according the type of vehicle actually being followed. However, Evans and Rothery (1976) found that different sized lead cars had no significant effect on following distance, while Parker (1996) measuring individual headways for a population of drivers passing through a roadworks section, found no significant differences between cars following cars as opposed to cars following trucks in a 6070kph speed band (approx. $25 \mathrm{~m}$ in each case), and only a small difference in a 20-30kph speed band (with cars being followed at approx. $12 \mathrm{~m}$, and trucks at $10 \mathrm{~m}$ ). In a 16 subject simulator test Yoo and Green (1999) found test participants followed a lead car some $10 \%$ closer than they did a truck ( $4.8 \mathrm{vs} .5 .3 \mathrm{sec}$ at $80 \mathrm{kph})$.

The clearest finding of direct relevance however is that due to Sayer, Mefford and Huang (2003), who analyzed the differential behavior between following cars and following light trucks from 1698 records collected as part of the Intelligent Cruise Control Operational Field Test (ICC FOT), which used 108 drivers for a minimum of two weeks each. Light trucks (on US roads at least) would seem to be followed some $6-7 \mathrm{~m}$ closer than normal cars at typical freeway speeds, approximately $0.22 \mathrm{sec}$. at $70 \mathrm{mph}$, in approximate agreement with our findings. Sayer et. al. (2003) provide a number of conjectures as to why this may be the case. Firstly, the 'big vehicles take longer to stop' hypothesis, which may be viewed as a combination of physical and sociological factors. For example it may be a popular belief that truck drivers (being professionals and having their livelihood depend on their driving, or more accurately, their accident avoiding skills) are less likely to misjudge any situation, anticipating and 'reading' the road far earlier and more accurately than any car driver. (A skill enhanced by their raised view point allowing them to see 'over' several of the vehicles ahead, and indeed necessary due to the deceleration characteristics of their vehicles). Truck braking frequency and indeed magnitude may therefore be perceived as being lower, leading car drivers to view them as 'safer' and more predictable vehicles to be behind. An alternate explanation suggested by the same authors is that a driver following a truck may have a reduction in driving workload as there is now only one vehicle on which to concentrate with resultant reductions in glance distributions. This gives the driver a greater degree of preparedness for deceleration, inturn encouraging an unconscious reduction in headway.

\section{iv) Drivers are inconsistent in their choice of headway.}

Confirmed: Evidence has been presented supporting individual (temporal) variations in adopted headway.

Our findings have demonstrated that if one considers comparable scenarios, controlling for the variables that we have considered, that in the majority of cases there is a significant change in headways between test runs with drivers in later runs exhibiting longer headways of 0.18 to 0.27 seconds. Such variations are likely to have implications to traffic stability and in near 
capacity flows. Unfortunately, even less quantitative research has been undertaken regarding this issue with the primary published finding of relevance being that of Lenne, Triggs and Redman (1997) who investigated variations in performance by using a driving simulator for tests on eleven subjects, with each using the simulator at one of six different times of day on different days. Between 10 am and 6 pm (i.e. roughly equivalent to the times used in this research) variations in average reaction times of approximately $12 \%$ were observed along with $8 \%$ variations in lateral position and almost $20 \%$ in speed variance. Although the core objective of Lennes research was to measure time of day variation, it is possible to argue that these results are also confounded with day-to-day variation, hence demonstrating, that significant changes in behavior can occur over time. If one extends this hypothesis to our data it is straightforward to calculate that the average percentage change in the headway between trials compared to the predicted value for our data is a little over $19 \%$.

Although not directly comparable, Hagland and Aberg (2002), in repeatedly measuring 75 drivers' free speeds on the same section of road on differing days, found a variation in free speed choice of on average $15 \%$. Despite this also being a choice, where the driver is continually trying to control the vehicle such that a dynamic variable is close to a subconscious target, in this case the 'target' (the desired speed) is far more difficult to perceive and consistently quantify compared to that of headway choice. For example while speed choice is hard to quantify without (occasional) reference glances to the speedometer, headway choice may be made in almost constant reference to the lead vehicle, for example the amount of road visible between the two vehicles or the angular size of the lead vehicle.

These findings aside there is little to go on, indeed even more generally in human factors as a whole the lack of research on 'test re-test' reliability is well known. For example, this was reviewed and highlighted by Lane, Kennedy and Jones (1986) who found little information on the area aside from tests on military personnel performing piloting and weapons tasks which demonstrated virtually no day to day correlation concluding from a review of the literature that "reliability across successive performances were rarely addressed or reported in either simulator of field studies". While this topic has been increasingly touched on in recent years (eg. Stanton and Young, 2003) in almost all cases, data analysed have been collected in artificial situations under which learning effects cannot be discounted, confounding the issue of true measure reliability.

Although usable data does now exist from large scale long-term field experiments such as the ICC FOT (Fancher et. al., 1998), and the more recent "100 car study" (Neale et. al., 2005), the data has yet to be examined with this objective in mind and although some analysis has been performed regarding driver adjustment to being observed (Dingus et. al., 2006, Chapter 8) and how behaviour changes through the use of a test vehicle (drivers would seem to adopt riskier behaviour), there is currently minimal data against which to compare our findings.

\section{CONCLUSIONS}

This paper has reported on an instrumented vehicle study of driver behavior in car following, focusing on factors affecting the choice of time headway. The study has collected over seven hours of time series data for six main test subjects driving on either motorway or high speed urban arterials with different levels of congestion. The data has been supplemented with five 
hours of data collected covertly on 125 drivers following the test vehicle. In addition to helping the ability to model congested roads (both theoretically and through simulation) this data and the conclusions of this analysis allows for more sophisticated design of advanced Intelligent Transport Systems (ITS), particular aiding in the design of in-vehicle based Advanced Driver Assistance Systems (ADAS).

The ecological validity of the data presented is hampered to an extent by the uncontrollability that is inherent in real road experiments. For example, it is possible to argue that in driving a test vehicle, the subjects may have been overly conservative, although there seems to be no consensus on this (Hjalmdahl and Varhelyi, 2004, Dingus et. al., 2006), or instructions to follow a confederate vehicle may encourage them to follow closer that they would do otherwise. While these are questions that have yet to be clearly resolved and are issues which apply to all instrumented vehicle, and indeed simulator, based experiments the scale of data collection within this research helps ensure the robustness of the findings produced.

By considering the day-to-day effect alongside a wide range of other explanatory variables this work has served to illustrate that a comparatively simple activity such as the following process is far more complex than may previously have been thought, containing a great deal of structure in what is frequently assumed to be simply random behavior. In summary, the main conclusions from this study are:

i) The level of flow does not seem in-itself to effect following headway choice.

ii) The road type (motorway versus urban dual carriageway) does not seem to affect headway choice.

iii) The type of lead vehicle does influence headway with drivers following closer to trucks/vans than they would to cars at the same speed.

iv) There is a small but distinct day-to-day effect within average headways for any individual driver.

Further studies will clearly be required to attempt to explain some of the features encountered, for example in terms of driver braking skill and experience (van Winsum and Heino, 1996) and indeed why drivers choose headways that are clearly less than their brake reaction times (an issue perhaps related to the lack of experience that most drivers have with anything more than low levels of braking, Touran et. al., 1999). However it is the last conclusion that has perhaps the greatest implication for further work in this field (and indeed all work on driver behaviour more generally), demonstrating that in future research consideration must also be given to longitudinal sampling effects. For example, consideration must be given to ascertaining how many 're-trials' of any one condition are necessary for measurements to converge to some kind of central mean with minimal variance, and casts doubt on the degree of credence that can be given to studies based on single trials, where, even in the absence of learning effects, driver motivation, mood (eg. aggressiveness or locus of control, Stokols, Novaco, Stokols and Campbell, 1978) and physiological factors may play major role in understanding the variability of results.

Such investigations are beyond the scope of this paper however, and will be examined as part of subsequent work where it is hoped that with the aid of cheaper more widely available data collection systems, such as those now seeing widespread use in for example, the ICC FOT (Fancher et. al., 1998) and the "100 car study" (Neale et. al., 2005), a database on a wider 
This is a post-peer-review, pre-copyedit version of an article published in Transportation Research Part $F$ : Traffic Psychology and Behavior. The definitive publisher-authenticated version - Brackstone $M$, Waterson B \& McDonald M (2009) "Determinants of Following Headway in Congested Traffic", Transportation Research Part F: Traffic Psychology and Behavior, 12(2), p131-142 - is available online at: http://dx.doi.org/10.1016/j.trf.2008.09.003

range of subjects will become available against which the issues raised in this paper may be further investigated.

\section{ACKNOWLEDGEMENTS}

Work reported in this paper has been funded by the EPSRC in the UK under contract GR/M94410 in the U.K., and was undertaken while the lead author was Senior Research Fellow at the University of Southampton. Part funding for the construction of the instrumented vehicle was supplied by the University of Southampton and Lucas-TRW. Grateful thanks are also given to: The Highways Agency and Owen Williams Consultants, for the provision of M27 flow data and the Southampton City Council 'ROMANSE' traffic control office for provision of A35 data. The authors are also indebted to Dr Beshr Sultan for helpful comments and suggestions during the preparation of this paper.

\section{REFERENCES}

- Allen, R.W., Magdeleno, R.E., Serafin, C., Eckert, S. and Sieja, T. (1997). Driver Car Following Behaviour Under Test Track and Open Road Driving Conditions. SAE Paper 970170. SAE.

- Aron, M., Biecheler, M. -B., Hakkert, S. A. and Peytavin, J. -F. (1997). Headways, RearEnd Collisions and Traffic: The Case of French Motorways. Proc. of the Int. Conf. On Traffic Safety on Two Continents, Lisbon, Portugal, 22-24, Sept., 1997. Paper 24/9. TRB, Washington D.C., U.S.A.

- Brackstone, M. (2000). An Examination of the Use of Fuzzy Sets to Describe Relative Speed Perception. Ergonomics, 43(4), 528-542.

- Brackstone, M. and McDonald, M. (2000). Car Following: A Historical Review. Transportation Research F, 2(4), 181-96.

- Brackstone, M., McDonald, M. and Sultan, B. (1999). Dynamic Behavioral Data Collection Using an Instrumented vehicle. Transpn. Res. Rec., 1689, 9-17.

- Brackstone, M., Sultan, B. and McDonald, M. (2002). Motorway Driver Behavior: Studies in Car Following. Transportation Research F. 5(1), 31-46.

- Chandler, R.E., Herman, R. and Montroll, E.W. (1958). Traffic Dynamics: Studies in Car Following. Opns. Res., 6, 165-184.

- Conchillo, A., Nunes, L., Recarte, M. and Ruiz, T. (1999). Speed Estimation in various Traffic Scenarios: Open Road and Closed Track. Vision in Vehicles VIII. Ed A Gale. Elsevier Science, Amsterdam. The Netherlands. In press

- Dijker, T., Bovy, P. H. L. and Vermijs, R. (1998). Car Following under Congested Conditions: Empirical Findings. Transpn. Res. Rec., 1644, 20-28.

- Dingus, T. A., Klauer, S. G., Neale, V. L., Petersen, A., Lee, S. E., Sudweeks, J., Perez, M. A., Hankey, J., Ramsey, D., Gupta, S., Bucher, C., Doerzaph, Z. R., Jermeland, J., and Knipling, R. R. (2006). .The 100-Car Naturalistic Driving Study, Phase II - Results of the 100-Car Field Experiment Performed by Virginia Tech Transportation Institute, Blacksburg, VA, Sponsored by National Highway Traffic Safety Administration, Washington, D.C. DOT HS 810593. 
- Dingus, T. A., McGehee, D. V., Manakkal, N., Jahns, S. K., Carney, C. and J. M. Hankey. (1997). Human Factors Field Evaluation of Automotive Headway Maintenance/ Collision Warning Devices. Human Factors, 39(2), 216-29.

- Evans, L. and Rothery, R. (1976). The influence of forward vision and target size on apparent inter-vehicular spacing. Transportation Science, 10, 85-101.

- Fancher, P., Ervin, R., Sayer, J., Hagan, M., Bogard, S., Bareket, Z., Mefford, M. and Haugen, J. (1998). Intelligent Cruise Control Field Operational Test (Final Report). Report DOT HS 808 849. National Highway Traffic Safety Administration, US DoT, Washington D.C.

- Hagland, M. and Aberg, L. (2002). Stability in Drivers' Speed Choice. Transportation Research F. 5(3), 177-188.

- Hjalmdahl, M. and Varhelyi, A. (2004). Validation of in-car observations, a method for driver assessment. Transportation Research A, 38, 127-142.

- Lane, N., Kennedy, R. and Jones, M. (1986). Overcoming unreliability in operational measures: The use of surrogate measure systems. Proceedings of the Human Factors Society 30th Annual Meeting, 1398-1402.

- Lenne, M., Triggs, T. and Redman, J. (1997). Time of Day Variations in Driving Performance. Accid. Analy. and Prev., 29(4), 431-437.

- Leutzbach, W. and Wiedemann, R. (1986). Development and Applications of Traffic Simulation Models at the Karlsruhe Institut fur Verkehrwesen. Traffic Engineering and Control, 27, 270-278

- Marsden, G., McDonald, M. and Brackstone, M. (2001). Towards an Understanding of Adaptive Cruise Control. Transportation Research C, 9(1), 33-51.

- Michael, P., Leeming, F. and Dwyer, W. (2000). Headway on Urban Streets: Observational Data and an Intervention to Decrease Tailgating. Transportation Research $F$, 3(1), 55-64.

- Neale, V., Dingus, T., Klauer, S., Sudweeks, J. and Goodman, M. (2005). An Overview of The 100-Car Naturalistic Driving Study and Findings, 19th International Technical Conference on Enhanced Safety of Vehicles (ESV), Washington, DC, June 6-9, 2005.

- Parker, M. T. (1996). The effect of Heavy Good Vehicles and Following Behavior on Capacity at Motorway Roadwork Sites. Traffic Engineering and Control, 37, 524-531.

- Postans, R. L. and Wilson, W. T. (1983). Close-following on the motorway. Ergonomics, 26(4), 317-327.

- Rajalin, S., Hassel, S. -O. and Summala, H. (1997). Close Following Drivers on Two Lane Highways. Accid. Analy. and Prev., 29(6), 723-729.

- Reiter, U. (1994). Empirical Studies as Basis for Traffic Flow Models. Proc. of the Second International Symp. on Highway Capacity. Sydney, Australia, Vol. 2, 493-502.

- Sayer, J. R., Mefford, M. L. and Huang, R. (2003). The effect of lead vehicle size on driver following behavior: Is ignorance truly bliss? Proc. of the $2^{\text {nd }}$ International Driving Symposium on Human Factors in Driver Assessment, Training and Vehicle Design. Park City, Utah, July 21-24. University of Iowa.

- Stanton, N. A. and Young, M. (2003). Giving ergonomics away? The application of ergonomics methods by novices. Applied Ergonomics, 34(5), 479-490.

- Stokols, D., Novaco, R. W. Stokols, J., and Campbell, J. (1978). Traffic Congestion, Type A Behavior, and Stress. Jnl. of Applied Psychology, 63(4), 467-480. 
- Sultan, B. (2000). The Study of Motorway Operation using a Microscopic Simulation Model. Doctoral Thesis, Dept. of Civil and Environmental Engineering, University of Southampton, U.K.

- Sumner, R., and Baguley, C. (1978). Close Following at two sites on rural 2 lane Motorways. Transportation Research Laboratory Report, LR859. Crowthorne, U.K.

- Taieb-Maimon, M. and Shinar, D. (2001). Minimum and Comfortable Driving Headways: Reality versus Perception. Human Factors, 43(1), 2001. 159-172.

- Treiber, M., Kesting, A. and Helbing, D. (2005). Delays, inaccuracies and anticipation in microscopic traffic models. Forthcoming in Physica A. [Preprint: cond-mat/0404736]. (http://arxiv.org/abs/cond-mat/?0404736).

- Touran, A., Brackstone, M. and McDonald, M. (1999). A Collision Model for Safety Evaluation of Autonomous Intelligent Cruise Control. Accid. Analy. and Prev., 31(5), 567-578.

- van der Hulst, M., Meijman, T. and Rothengatter, T. (1998). Anticipation and the Adaptive Control of Safety Margins in Driving. Ergonomics, 42(2), 336-345.

- van Winsum, W. and Heino, A. (1996). Choice of time headway in car following and the role of time to collision information in braking. Ergonomics, 39, 579-592.

- Yoo, H. and Green, P. (1999). Driver Behavior while Following Cars, Trucks and Buses. Report No. UMTRI-99-14. Ann Arbor, MI. The University of Michigan Transportation Research Institute. 